\title{
Aproximación al diseño conceptual y estructural del viaducto de Almonte
}

\section{Approach to the conceptual and structural design of the viaduct over River Almonte}

\author{
Guillermo Capellán Miguel ${ }^{\mathrm{a}}$ y Pascual García Arias ${ }^{\mathrm{b}}$ \\ ${ }^{a}$ Dr. Ingeniero de Caminos, Canales y Puertos. Arenas \& Asociados, Santander, España \\ ${ }^{b}$ Ingeniero de Caminos, Canales y Puertos. IDOM Consulting, Engineering, Architecture, Madrid, España
}

Recibido el 18 de diciembre de 2018; aceptado el 22 de febrero de 2019

\section{RESUMEN}

El viaducto de Almonte, con su gran arco de $384 \mathrm{~m}$ de luz para el nuevo ff.cc. de alta velocidad, ha supuesto un verdadero reto técnico desde el punto de vista del proyecto y la construcción. Desde un primer momento, Juan José Arenas tiene claro que, dadas las condiciones de trazado, orografía y cimentación sobre roca, la solución adecuada es un puente arco de hormigón. Porque el arco resistiendo por forma, es la manera más eficiente, segura y rígida para realizar la estructura, pero también por un compromiso ineludible con la durabilidad.

(C) 2020 Asociación Española de Ingeniería Estructural (ACHE). Publicado por Cinter Divulgación Técnica S.L.L. Todos los derechos reservados. PALABRAS CLAVE: arco, hormigón, alta velocidad, antifunicularidad, línea de presión.

\section{ABSTRACT}

The Viaduct over River Almonte, with its $384 \mathrm{~m}$ arch for the new high-speed rail, has been a real technical challenge from the point of view of design and construction. From the very beginning, Juan José Arenas has in mind that, given the conditions of layout, orography and foundation on rock, the right solution is a concrete arch bridge. Because the arch resisting by form, is the most efficient, safe and rigid way to make this structure, but also because of an unavoidable commitment to durability.

(C) 2020 Asociación Española de Ingeniería Estructural (ACHE). Published by Cinter Divulgación Técnica S.L.L. All rights reserved.

KEYWORDS: arch, concrete, high speed, antifunicular directrix, line of thrust.

1.

\section{LOS INICIOS DE UN PROYECTO}

Cada administración es responsable de seleccionar los equipos técnicos adecuados para desarrollar los proyectos que precisa construir. En el caso de la Línea de Alta Velocidad Madrid-Extremadura, ADIF seleccionó en concurso al equipo IDOMArenas \& Asociados, para encargarse del proyecto constructivo del tramo que incluía el salto sobre el río Almonte en una de las colas del embalse de Alcántara.

Con esa selección ya tomó una importante decisión sobre la calidad, seguridad y durabilidad que quería aplicar a un reto tan importante. Había puesto al equipo de IDOM y al de Arenas \& Asociados, con Juan José Arenas y todo su importante

\footnotetext{
* Persona de contacto / Corresponding author:

Correo-e / email: gcapellan@arenasing.com (Guillermo Capellán).
}

bagaje, a la cabeza del diseño y proyecto del puente sobre el Almonte.

Así, en las primeras y decisivas reuniones mantenidas en las oficinas de ADIF con los responsables del proyecto Juan José Llamas, Fidel Corral e Ignacio Meana, se analizaron las posibilidades de cruce de los puentes del Tajo, Almonte y resto de viaductos del tramo, en presencia de los diseñadores Javier Manterola, Carlos Siegrist, J.J. Arenas y sus respectivos colaboradores.

Es en esa fase de análisis, debate y discusión, en la que se le pregunta a Juan José Arenas “¿qué opina el Profesor...?", a lo cual, tras un pequeño silencio, contestó con seguridad: "Yo prefiero moverme en el dominio del arco". 


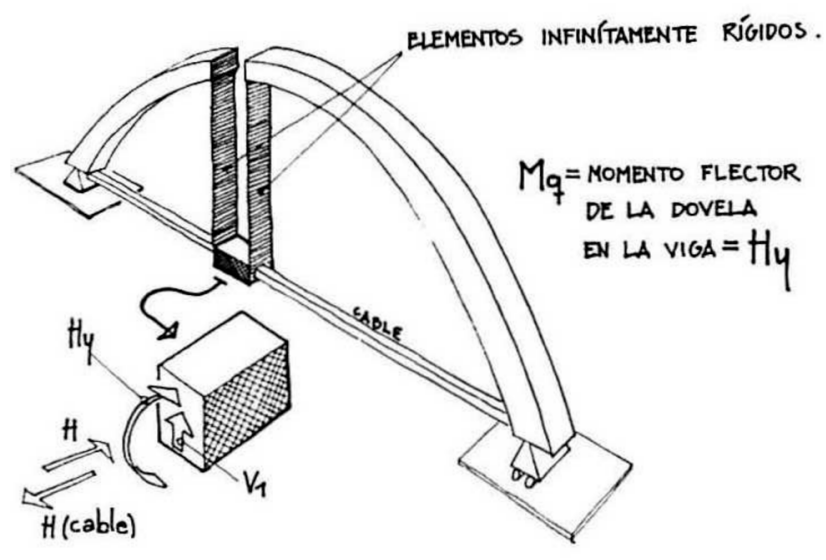

Figura 1. Representación de esfuerzos en dovela de arco y viga equivalente [1].

La frase parecía sencilla y quizás intrascendente, pero en ella estaba implícita la correcta lectura del cruce con su perfil en $\mathrm{V}$, las condiciones de cimentación de las laderas y la altura del cruce de la línea sobre el río, que permitían aprovechar el espacio y volumen para encajar sin miedo un puente adecuado. Es decir, se piensa que, teniendo adecuadas condiciones de contorno, si se recurre al arco en una situación de "libertad mecánica", se podrá dar buena respuesta estructural al reto de dar soporte a una línea de alta velocidad a $320 \mathrm{~km} / \mathrm{h}$ para luces extraordinarias.

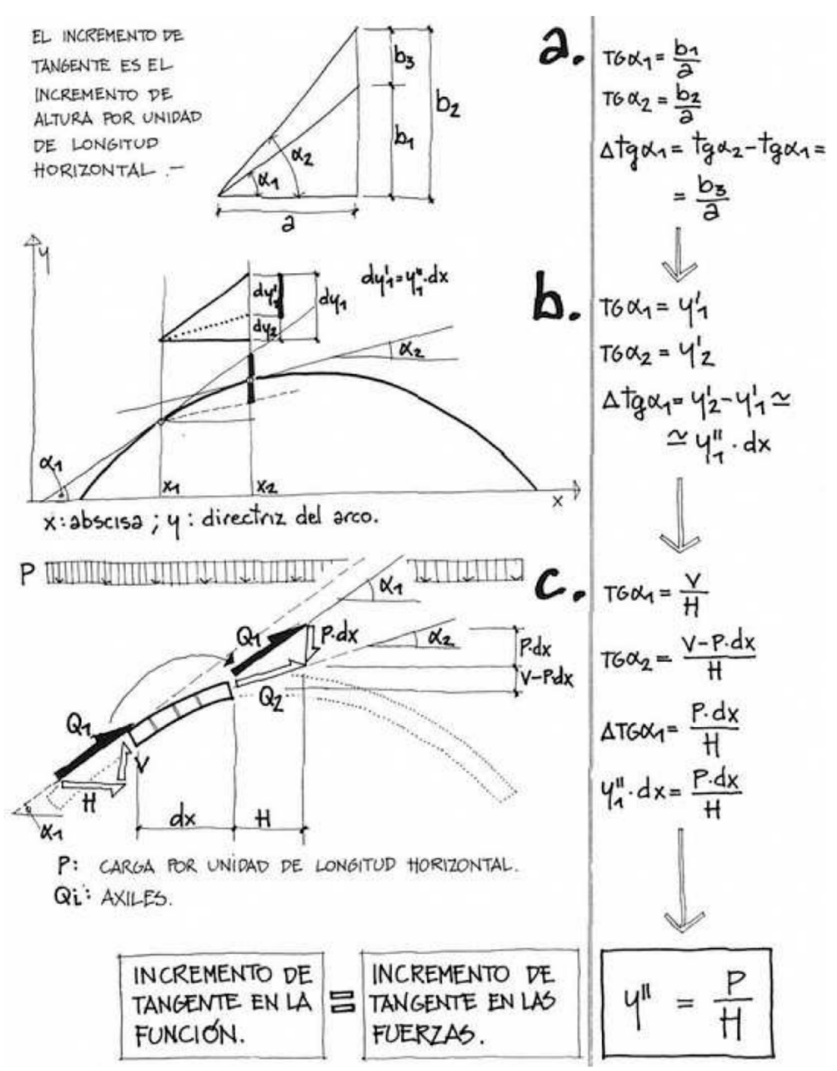

Figura 2. Expresión matemática de la condición de antifunicularidad en un arco [1].

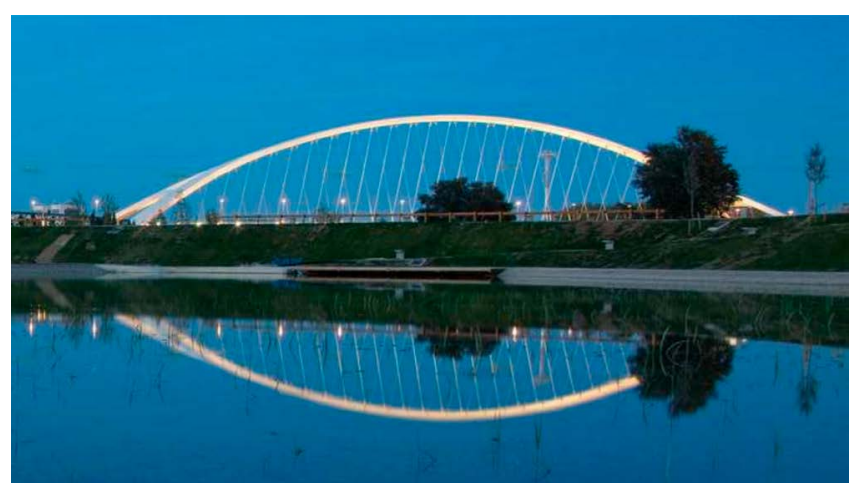

Figura 3. La esbeltez como aplicación rigurosa de la antifunicularidad. Puente del Tercer Milenio, Zaragoza.

Con ello, Arenas estaba queriendo moverse en una zona de relativa comodidad en la que pese a las importantes exigencias deformacionales, vibratorias y dinámicas que impone el ferrocarril de altas prestaciones, pensaba contar con la ayuda del arco y su trabajo por forma para responder a las demandas de las cargas (figura 1).

Juan José Arenas, al decir aquella frase, estaba llevando el problema a su espacio de confort, que buenos resultados le había dado en puentes como La Barqueta, Oblatas, La Regenta, Morlans o el Puente del Tercer Milenio. Es probable que proyectistas con diferente trayectoria hubiesen tratado el problema de otra forma, pero siempre en Juan José quedaba, y pesaba en el fondo, algo de lo que Carlos Fernández Casado decía en sus notas esenciales de la estructura arco, en su libro de Cálculo de Arcos: "el arco tiene la pretensión de ser configuración de esfuerzos, es decir, funicular de las fuerzas aplicadas. El grado mayor o menor en que esto se logra define la perfección de la estructura" [2].

Así, la búsqueda de esa perfección estructural no es la de la verdad estructural, ni un grial de las estructuras, sino la de la máxima conveniencia económica y funcional dando lugar a una adecuación mecánica en donde se atiende a lo estructural como prioritario; buscar con la geometría la antifunicularidad, es decir, "pegar" la materia a la línea de presión, al camino de las cargas (figura 2).

Bien se tenía en nuestro equipo la experiencia, sobre todo tras el Puente del Tercer Milenio (figura 3), que el arco si es trazado elegante y encajado esbelto con mínima cantidad de materia por volumen, fruto de una directriz antifunicular, será estéticamente positivo.

Así que aquí, otra vez, forma y función son una misma cosa siguiendo una fórmula que ha sido hilo conductor en todas las obras de Juan José Arenas y su equipo, en las que la coherencia estructural, la conveniencia y la economía han sido aplicación del funcionalismo moderno.

De este modo, ante un reto estructural nuevo, se aplica una solución que descarta riesgos o excursiones formales, y atiende prioritariamente a la técnica; pero como en toda la trayectoria anterior, se aplica sensibilidad y creatividad a la formalización y materialización del concepto estructural. Y no sólo al ordenamiento de los elementos estructurales y de sus formas y proporciones, sino a su composición general, escala y encaje en el paisaje, junto al cuidado de los detalles aislados que articulan la relación entre ellos. 
La visión del puente como parte del territorio y como consecuencia de un acto humano está presente en toda la obra, siguiendo el título del discurso que J.J. Arenas produjo en 1982 como lección inaugural del curso académico de la Universidad de Cantabria: "El Puente, pieza esencial del mundo humanizado" [3].

Todo ello estaba, ya implícito en su frase "me gustaría moverme en el dominio del arco".

\section{2.}

\section{PRINCIPIOS FUNDAMENTALES DEL DISEÑO}

Una vez lograda la aceptación general de la tipología arco como la más adecuada en términos de eficiencia estructural, durabilidad y mantenimiento al no "tener costuras", Juan José y su equipo toman una serie de decisiones clave en el diseño del viaducto de Almonte para convertirlo en vanguardia del determinismo tecnológico.

El diseño de la directriz del arco, es una de las claves principales del diseño. No es una parábola como en los puentes de carretera, en los que el peso propio de la estructura es muy superior al de la sobrecarga, y por tanto estas no desvían la línea de presión fuera del núcleo central. En puentes metálicos en los que las sobrecargas desvían la línea de empujes respecto a la teórica antifunicular de peso propio, se suele adoptar la geometría antifunicular para el 50\% de las sobrecargas.

Sin embargo, en un puente ferroviario, tenemos mucho peso propio si es de hormigón, pero también sobrecargas muy importantes que inducen grandes desviaciones de la línea de empuje. La estrategia novedosa, es calcular la directriz antifunicular para la sobrecarga amplificada por el comportamiento dinámico de los trenes de alta velocidad. Es decir, para los trenes HS-A y HS-M se realiza un cálculo dinámico y se ajusta iterativamente la directriz y el canto del arco para asegurar que la línea de presiones permanece dentro del núcleo central para la combinación característica (ELS) en todas las secciones de la directriz.

Debido a la extraordinaria luz del arco y a la reducida sección del tablero, típica en puentes de alta velocidad, la rigidez transversal del conjunto es reducida frente a cargas horizontales. Un reto del diseño era, por tanto, la limitación de las deformaciones y vibraciones del puente. En ello juega un papel esencial la sección transversal del arco por la necesidad de reducir las fuerzas resultantes de la acción del viento. Por ello se diseña una sección con biseles pronunciados que evitan la clásica sección rectangular, y que como se demuestra en el túnel de viento, consiguen una reducción significativa de la presión de viento sobre dicha sección.

Para verificar la bondad de las secciones y la mejora del comportamiento global de la estructura desde el punto de vista aerolástico, se desarrolló un ensayo completo en túnel de viento de capa límite que permitió adecuar las secciones del viento reales y verificar la ausencia de resonancias y la magnitud de las vibraciones horizontales, que no suelen ser acotadas por los códigos de diseño.

Un arco de la luz proyectada supone una gran esbeltez fuera del plano vertical, por lo que los criterios de inestabilidad en dicha dirección deben garantizar la singularidad estructural.
Más allá de aumentar el ancho del cajón hasta valores significativos que además suponen incremento del peso (y por tanto coste y medios auxiliares), la estrategia de diseño consistió en separar el arco en dos patas que permiten mantener el peso por metro del arco uniforme y aumentar mucho la rigidez transversal del conjunto de forma que el modo de pandeo principal es el propio plano y no fuera de él. Esto, unido al diseño de la sección transversal, permite maximizar la rigidez transversal y minimizar los empujes del viento, dotando al conjunto de un comportamiento excelente fuera del plano a través del diseño.

Estos tres factores son los principios básicos sobre los que se sustenta el diseño singular del viaducto de Almonte.

Pero tampoco se olvida la imponente tradición española de puentes arco, en este caso de hormigón. Si en 1942, Martín Gil junto a Eduardo Torroja nos legaban el imponente viaducto sobre el Esla, desde que Juan José Arenas retomara esta tipología clásica con el Arco de La Regenta (1996, 194 m de luz, APIA XXI), los proyectistas españoles han dejado una huella excelsa a lo largo de toda la península Ibérica. El Burguillo (1999, 165 m, FHECOR), el Arco del Infante Henrique (2002, 280 m, IDEAM+Adão da Fonseca), Cieza (2004, 141 m, Pondio+Siegrist y Moreno), Los Tilos (2004, 255 m, Ferrovial-Agromán), el Almonte carretero (2005, 184 m, Siegrist y Moreno), Contreras (2009, 261 m, CFCSL), o el Ulla (2010, 168 m, Torroja), constituyen un conjunto difícilmente superable, y nutren de confianza y conocimiento a las constructoras españolas que los ejecutaron.

Todos y cada uno se tienen en cuenta, se estudian y analizan de manera pormenorizada, para conseguir un proyecto lo más eficiente posible. Y unido a ello, el equipo de diseño se apoya en la amplia experiencia española en puentes de alta velocidad, en busca de la mayor optimización en la construcción del nuevo puente sobre el Almonte.

El buen uso de la autocimbra en la construcción de los tableros de la amplia colección de viaductos de gran porte en los accesos de alta velocidad a Galicia, en la que Arenas \& Asociados participa de su supervisión junto a IDOM, lleva a concebir vanos de acceso de $45 \mathrm{~m}$, adaptados a la tecnología del momento, en busca de una construcción del tablero lo más estandarizada y simplificada posible.

Pero además, la exitosa construcción del arco del viaducto de Contreras, proyecto de CFCSL, por atirantamiento provisional desde las pilas adyacentes al mismo, lleva a considerar este procedimiento constructivo como preferente por su buen comportamiento estructural y su mínima afección al Embalse de Alcántara.

En cuanto al punto fijo de la estructura, se decide disponerlo en la clave del arco, planteamiento radicalmente opuesto a lo desarrollado en el resto de la red de alta velocidad española donde se ubica en estribos, disminuyendo la distancia de dilatación en la mitad y simplificando por tanto los aparatos de dilatación de vía y juntas de expansión necesarios.

Ahora que las obras están terminadas es posible observar la magnitud de esta estructura que no transmiten por completo las fotografías, y que solo podía imaginarse durante la redacción del proyecto.

Un proyecto concebido, desarrollado y finalmente construido por un magnífico grupo de profesionales de muy distintas empresas, sin el que la obra que hoy en día se reconoce internacionalmente hubiese sido posible. Gracias a todos ellos. 


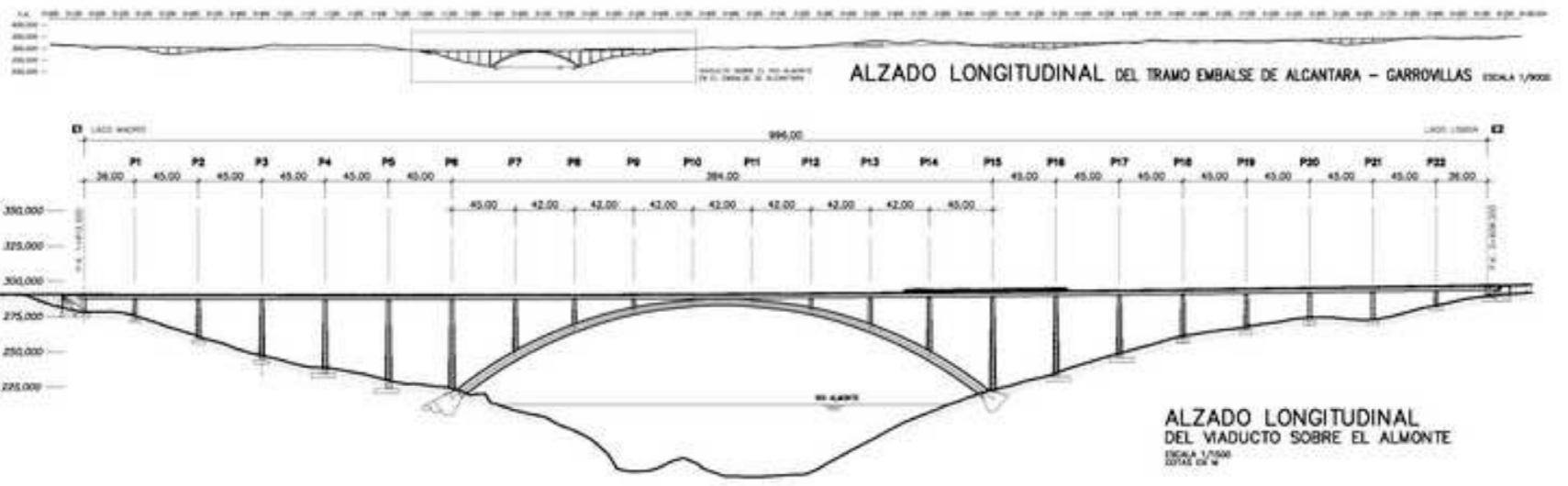

Figura 4. Alzado del viaducto de Almonte.

3.

\section{LA CONCEPCIÓN DEL PUENTE}

La línea de Alta Velocidad Madrid-Extremadura cruza sobre el río Almonte en la cola del embalse cacereño de Alcántara, mediante el viaducto de Almonte, de $996 \mathrm{~m}$ de longitud. Sobrevuela el embalse por medio de un gran arco de hormigón de tablero superior y $384 \mathrm{~m}$ de luz principal, que lo convierten en uno de los puentes arco de alta velocidad con mayor luz del mundo (figura 4).

El puente forma parte del tramo Embalse de Alcántara Garrovillas dentro de la línea destinada en origen y a futuro para conectar Madrid y Lisboa mediante ferrocarril de Alta Velocidad, prevista para tráfico mixto de pasajeros y mercancías, con velocidad máxima de hasta $350 \mathrm{~km} / \mathrm{h}$.

La restricción impuesta para este viaducto fue la de no disponer pilas en el cauce del embalse, y los corredores de cruce posible venían marcados por la Declaración de Impacto Ambiental.

Dichas imposiciones, condujeron al vano de $384 \mathrm{~m}$, lo que sitúa los arranques del arco justo por encima del nivel máximo de las aguas del embalse (cota $218 \mathrm{~m}$ ), que en ningún caso podía alterar su funcionamiento como aprovechamiento hidroeléctrico por el desarrollo de las obras. Durante buena parte de las mismas hemos visto el embalse en su nivel máximo a punto de desbordar las penínsulas provisionales de protección realizadas para la ejecución de las cimentaciones del arco, ratificando la elección de la luz adoptada como la mínima para no depender del embalse.

Tanto el diseño como el proceso constructivo elegido para materializar el mismo, buscan desde el comienzo del proyecto conjugar la viabilidad técnica y la optimización de medios y costes de una actuación tan singular como esta [4].

El viaducto está localizado entre los puntos kilométricos $1+612.200$ y $2+608.200$ y se sitúa en una recta en planta. En alzado existe un acuerdo vertical que genera un punto bajo en el punto kilométrico $1+815.198$ siendo la pendiente máxima del viaducto de 14.6 milésimas.

La rasante alcanza una altura máxima sobre el río Almonte de unos $80 \mathrm{~m}$. Esto permite el encaje de un arco de altura 69 $\mathrm{m}$, es decir una relación clásica de flecha/luz de 1:5.6. Todo ello, una vez fue seleccionada la tipología de puente arco como la más adecuada.
Así, la infraestructura cuenta con veintitrés vanos de tablero ejecutados con autocimbra superior, con luces tipo de $45 \mathrm{~m}$ entre pilas en las zonas que discurren fuera del arco y de $42 \mathrm{~m}$ sobre las pilastras que apoyan directamente sobre el arco.

4.

\section{CONFIRMACIÓN DE LA TIPOLOGÍA}

Durante la redacción del proyecto, a pesar de lo relatado en el punto primero que indica la preferencia por el arco desde los estadios iniciales, se analizaron distintas variantes estructurales en un estudio tipológico detallado considerando de forma simultánea su comportamiento final, así como el procedimiento constructivo.
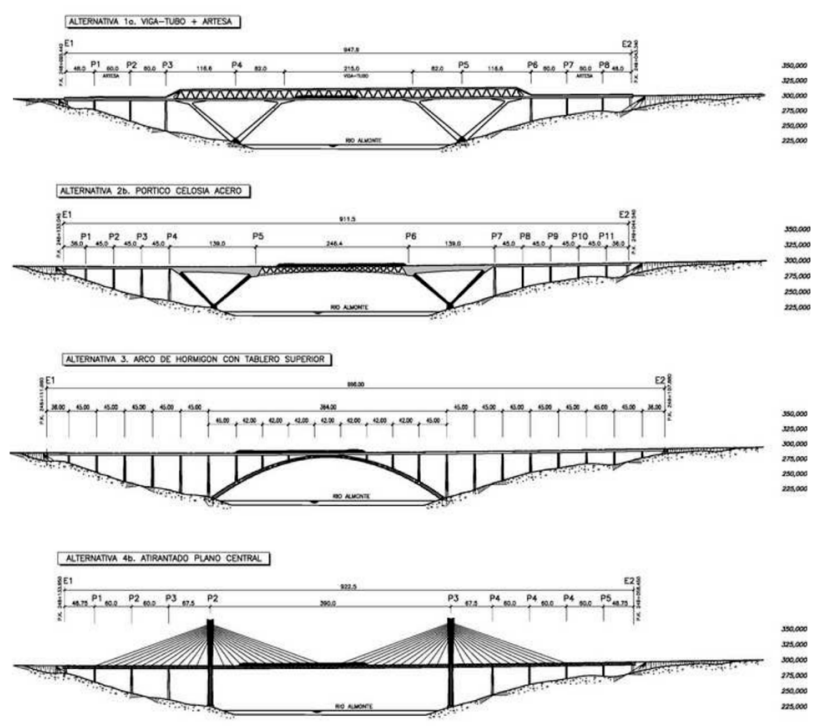

Figura 5. Diferentes alternativas consideradas en el estudio de tipologías.

Algunas de estas alternativas incluían soluciones atirantadas, de tipo pórtico, y tableros de tipo viga celosía de canto variable (figura 5). Las soluciones de procedimiento constructivo 
incluían distintas variantes de avance en voladizo, la erección de un tramo central metálico, etc.

Realizado el estudio tipológico, se decidieron los principales factores de evaluación y ponderación de las alternativas:

- Industrialización del proceso: se pretendía evaluar las dificultades constructivas planteadas por cada solución, penalizando aquellas en las que podían presentarse con más probabilidad incidencias que afectasen al plazo de ejecución.

- Coste de inspección y mantenimiento: factor a tener en cuenta para cualquier estructura, e íntimamente ligado a la durabilidad de la misma.

Especialmente importante en puentes poco accesibles es la necesidad o no de prever reparaciones y operaciones de mantenimiento periódicas como sucede en las estructuras metálicas y atirantadas.

- Coste de ejecución: atendiendo a la valoración económica realizada para cada una de las distintas soluciones, se valoró de forma negativa las que suponían un mayor coste de las obras.

- Plazo de ejecución: a partir de una estimación de rendimientos, se ponderaron las soluciones, siendo las menos penalizadas aquellas que conllevaban un menor plazo de ejecución.

- Calidad estética de la solución: si bien es claro que toda alternativa planteada, cuidadosamente trabajada, ha de conducir a una buena calidad estética de la solución, se considera que por su propia tipología hay alternativas que tienen más belleza formal.

- Encaje medioambiental de la solución: este apartado se disocia del punto anterior, porque con él no se valora la calidad de una solución en sí misma, sino en relación con el entorno natural y las condiciones faunísticas en las que se ubica.
- Mejoras e innovación: se valoran especialmente positivas las soluciones que presentan diseños orientados a la reducción de presiones de viento y mejora aerodinámica.

La problemática de las vibraciones transversales en tableros largos de gran luz es todavía poco conocida. Soluciones con forma aerodinámica o especial rigidez transversal se adaptan bien al problema de cruce del valle del río Almonte.

Así, el análisis multicriterio llevado a cabo, destacó la solución arco como la más económica, la de mejores condiciones de durabilidad y mantenimiento, y la que garantizaba un mejor comportamiento estructural frente a fenómenos dinámicos y de viento, como ya vaticinaba el Profesor Arenas.

Esta preocupación por la durabilidad ha sido una constante durante el proceso de proyecto y ejecución de las obras, buscando legar una obra que resista de la mejor forma el paso del tiempo, con el menor mantenimiento. Para ello la elección del hormigón, la geometría del arco, el desarrollo de los cálculos y la definición del procedimiento constructivo en ausencia de fisuraciones, resulta definitivo. La elección de la tipología tiene también mucha relación con los condicionantes no solo del emplazamiento, con la elevada altura de rasante y buenas condiciones de cimentación directa en roca, sino de las propias peculiaridades de los puentes ferroviarios de alta velocidad, en este caso de gran luz.

Las particularidades que se dan en estos puentes son unas cargas de tráfico mucho mayores que las de carretera, unos efectos dinámicos por el paso de los convoyes, unas cargas horizontales notables y un trabajo de fatiga que no se puede despreciar. Todo esto debe conjugarse con unas consideraciones funcionales muy estrictas para que el nivel de servicio de la estructura no deba limitarse en ningún momento: deflexiones y aceleraciones permisibles pequeñas y una longitud entre juntas de dilatación de vía limitada por motivos tecnológicos.

TABLA 1

Estudio comparativo de soluciones - Tipología y definición de estructuras del proyecto de construcción (Fuente: ADIF AV).

\begin{tabular}{|c|c|c|c|c|c|c|c|c|c|c|c|c|c|c|c|}
\hline \multirow{3}{*}{$\begin{array}{l}\text { Condicionante } \\
\text { Industrialización } \\
\text { del proceso }\end{array}$} & \multirow{3}{*}{$\begin{array}{c}\text { Factor } \\
2\end{array}$} & \multicolumn{2}{|c|}{ la } & \multicolumn{2}{|c|}{$1 b$} & \multicolumn{2}{|c|}{$2 \mathrm{a}$} & \multicolumn{2}{|c|}{$2 b$} & \multicolumn{2}{|c|}{3} & \multicolumn{2}{|c|}{$4 a$} & \multicolumn{2}{|c|}{$4 b$} \\
\hline & & $\begin{array}{l}\text { Vig } \\
+\mathrm{a}\end{array}$ & $\begin{array}{l}\text { tubo } \\
\text { tesa }\end{array}$ & Vig & tubo & $\begin{array}{r}\text { Pórtice } \\
\text { horn }\end{array}$ & $\begin{array}{l}\text { celosía } \\
\text { iigón }\end{array}$ & $\begin{array}{r}\text { Pórticc } \\
\text { met }\end{array}$ & $\begin{array}{l}\text { celosía } \\
\text { lica }\end{array}$ & $\begin{array}{r}A \\
\text { hor }\end{array}$ & $\begin{array}{l}\text { o } \\
\text { igón }\end{array}$ & $\begin{array}{l}\text { Atir } \\
\text { dobl }\end{array}$ & $\begin{array}{l}\text { tado } \\
\text { plano }\end{array}$ & $\begin{array}{l}\text { Atirar } \\
\text { plano }\end{array}$ & $\begin{array}{l}\text { tado } \\
\text { entral }\end{array}$ \\
\hline & & 1 & 2 & 1 & 2 & 4 & 8 & 3 & 6 & 3 & 6 & 2 & 4 & 2 & 4 \\
\hline Coste de ejecución & 5 & 4 & 20 & 5 & 25 & 2 & 10 & 4 & 20 & 1 & 5 & 2 & 10 & 3 & 15 \\
\hline $\begin{array}{l}\text { Mantenimiento } \\
\text { y durabilidad }\end{array}$ & 3 & 2 & 6 & 2 & 6 & 2 & 6 & 2 & 6 & 1 & 3 & 3 & 9 & 3 & 9 \\
\hline Plazo de ejecución & 2 & 2 & 4 & 2 & 4 & 3 & 6 & 2 & 4 & 2 & 4 & 1 & 2 & 1 & 2 \\
\hline Calidad estética & 1 & 4 & 4 & 4 & 4 & 3 & 3 & 3 & 3 & 1 & 1 & 2 & 2 & 1 & 1 \\
\hline $\begin{array}{l}\text { Encaje } \\
\text { medioambiental }\end{array}$ & 2 & 1 & 2 & 1 & 2 & 1 & 2 & 1 & 2 & 1 & 2 & 2 & 4 & 2 & 4 \\
\hline Mejoras e innovación & 2 & 2 & 4 & 2 & 4 & 2 & 4 & 2 & 4 & 1 & 2 & 3 & 6 & 4 & 8 \\
\hline
\end{tabular}

TOTAL 
Dadas esas particularidades, las luces mayores de $100 \mathrm{~m}$ hasta ahora han sido inusuales en los puentes para ferrocarril de alta velocidad y, por tanto, la luz que ha sido necesario adoptar para salvar el Embalse de Alcántara entra dentro de un rango excepcional.

A esto se une en el caso del viaducto del Almonte, que transcurre a una altura de más de $80 \mathrm{~m}$ sobre la lámina de agua y con altura de pilas de hasta $65.3 \mathrm{~m}$, lo que acentúa la preocupación por los efectos aerolásticos y aerodinámicos de viento y las deformaciones transversales bajo estas y el resto de cargas horizontales.

El arco es una estructura de baja deformabilidad vertical, no en vano el canto efectivo del sistema estructural o la "viga equivalente" es la flecha del arco, es decir $69 \mathrm{~m}$, en este caso. Para limitar la deformabilidad lateral y mejorar su comportamiento a viento se actúa definiendo una sección octogonal del arco aerodinámica frente al viento, buscando la mayor esbeltez del arco, y abriendo en transversal su sección en arranques en dos pies inclinados curvos que aumentan el brazo resistente en esta dirección (figura 6).

\section{5. DESCRIPCIÓN DE ELEMENTOS DE LA ESTRUCTURA}

El ancho total del tablero es de 14.0 metros. La sección trasversal del tablero está formada por 10.1 metros centrales para alojar el balasto de la plataforma, dos muretes guardabalasto de $0.20 \mathrm{~m}$ y espacios laterales para alojar impostas, canaletas, paso de servicio, barreras y protecciones de aves.

Se trata de una estructura de tablero hiperestático, con sección cajón de hormigón pretensado ejecutado in situ. La construcción se plantea en fases sucesivas, mediante autocimbra. Su configuración es la de un tablero estándar para puentes multivano de alta velocidad de vanos tipo de $45 \mathrm{~m}$ en accesos ejecutado con autocimbra, lo que colabora a la reducción de costes y mejora de rendimientos en su ejecución, pudiendo extender el mismo procedimiento constructivo a todo lo largo del tablero, incluso sobre el arco sobre el embalse, solo reduciendo el vano tipo a $42 \mathrm{~m}$ para adaptarse a la longitud del vano y las diferentes condiciones de apoyo.

El tablero se resuelve mediante una sección cajón de hormigón pretensado de canto constante 3.10 metros (relación luz/canto 1/15). Los voladizos del cajón son de 3.25 metros y el ancho de la parte superior del núcleo del cajón es de 7.50 metros. La anchura del fondo del núcleo es de 6.00 metros.

El pretensado de los vanos de acceso está formado por 4 familias de 3 tendones ( 6 tendones por alma) de 27 cordones de $0.6^{\prime \prime}$. En los vanos sobre arco se mantienen estas 4 familias de tres tendones ( 6 por alma) pero con 31 cordones de $0.6^{\prime \prime}$. Además, se disponen familias adicionales sobre pila o en centro de vano como refuerzo en tramos principales sobre el arco.

El arco, de hormigón de alta resistencia HA-80 autocompactable, tiene una sección octogonal hueca en sus 210 $\mathrm{m}$ centrales, bifurcándose a continuación en dos pies de sección hexagonal irregular también hueca hasta sus arranques, para dotarlo de mayor estabilidad transversal. Ambos pies se arriostran entre sí en el empotramiento entre la segunda pila (comenzando desde los arranques) y el arco. La sección octo- gonal tiene un comportamiento aerodinámico adecuado, muy beneficioso en grandes luces como la que estamos considerando. La sección varía entre los $6 \mathrm{~m}$ de ancho central y canto de $4.80 \mathrm{~m}$, y un ancho total de $19 \mathrm{~m}$ entre ambos pies y $6.90 \mathrm{~m}$ de canto, lo que supone una relación luz/canto variando entre $1 / 80$ y $1 / 56$.

Las pilas del viaducto, tanto las que se cimientan sobre el terreno como las que se apoyan en el arco (también denominadas montantes o pilastras), tienen sección octogonal variable, cuya aerodinámica es beneficiosa en el vano del arco dada su gran luz, que se mantiene en las pilas de los vanos de acceso para mantener el equilibrio visual del conjunto. La altura de pilas alcanza los $65.3 \mathrm{~m}$.

Los estribos son de hormigón armado, cerrados con aletas en vuelta.

El apoyo del tablero sobre pilas y estribos se materializa mediante apoyos de neopreno confinado tipo "POT" con teflón en parejas de apoyos libres y guiados con restricción transversal.

El punto fijo se sitúa en el centro del arco, materializado mediante una unión entre el arco y tablero que permite transmitir los esfuerzos horizontales de frenado y arranque al arco. De esta forma las longitudes de movimientos horizontales debidas a las variaciones de temperatura y reología del hormigón que han de asumir los estribos, son los correspondientes a 450 y $546 \mathrm{~m}$ respectivamente, perfectamente asumibles.

Las cimentaciones de arco, pilas de vanos de acceso y estribos se resuelven mediante zapatas sobre el sustrato rocoso. Cada arranque del arco y su pila adyacente cuentan con una cimentación conjunta. Las cimentaciones de los arcos son unos grandes macizos cajeados en la roca cuya ejecución requiere de penínsulas provisionales de protección para bajar por debajo del nivel máximo de aguas del embalse.

\section{6. \\ CÁLCULO ESTRUCTURAL E INFLUENCIA DEL ENCAJE DE LA DIRECTRIZ DEL ARCO EN EL DISEÑO DEL ARCO}

A consecuencia de la naturaleza de la propia estructura, de las condiciones de servicio, y del método constructivo el análisis estructural debe incluir:

- El Estudio de la directriz óptima del arco en base al cálculo de la antifunicular de las cargas.

- El Análisis detallado de las fases constructivas.

- La valoración de estado límite de servicio y último con modelos de cálculo no lineales.

- El análisis dinámico detallado.

- Entre los estudios especiales se ha de señalar la confirmación de los resultados de los estudios analíticos realizados del comportamiento de la estructura frente a las cargas de viento, mediante la realización de estudios experimentales con ensayo en túnel de viento.

En el diseño de puentes arco la búsqueda de la geometría de su directriz es un punto clave del diseño, ya que si es la adecuada trabajará por forma, es decir, sometido solo a tensiones normales de compresión en todos y cada uno de sus puntos, funcionando como antifunicular de las cargas. 


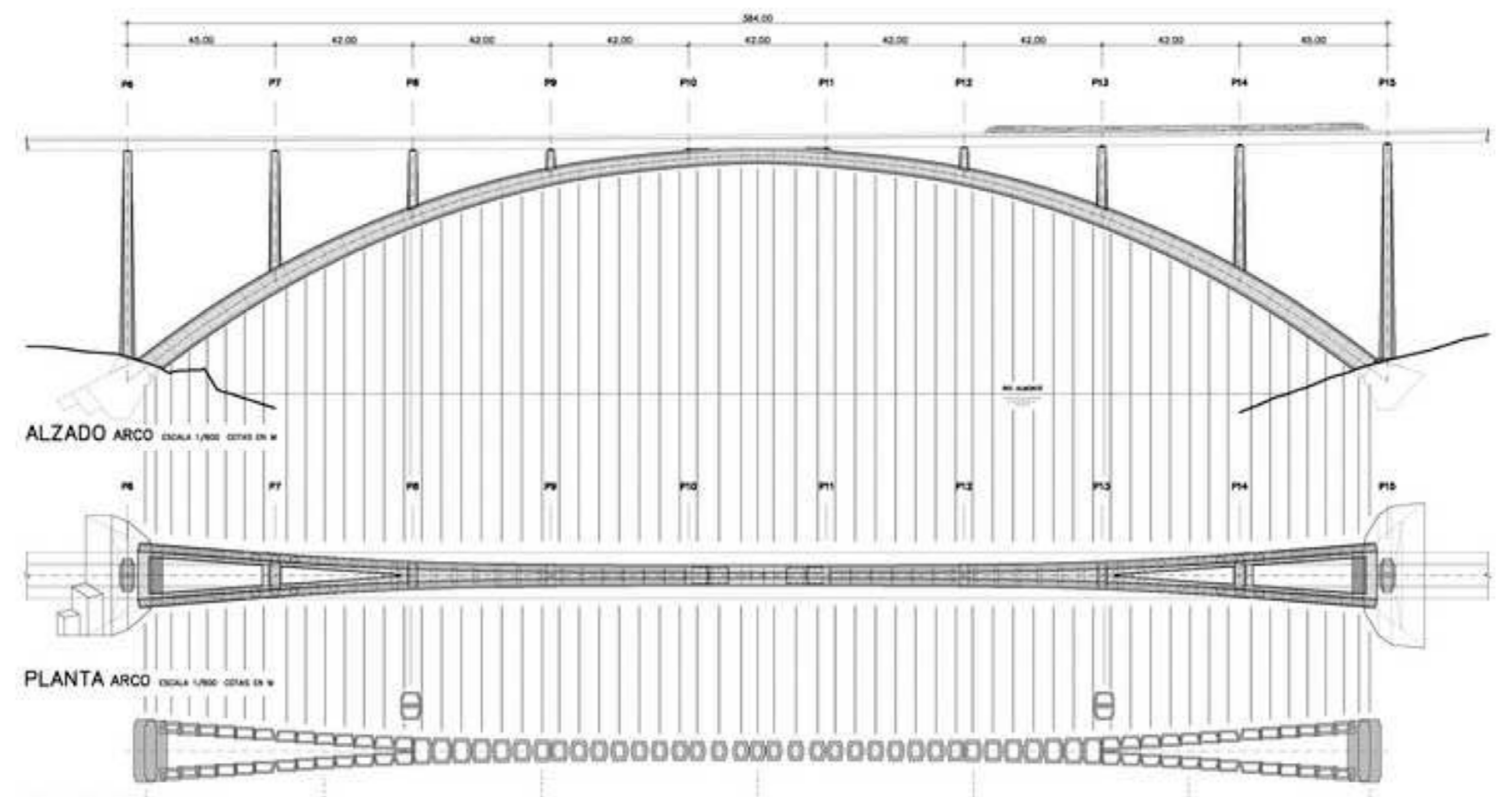

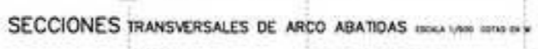

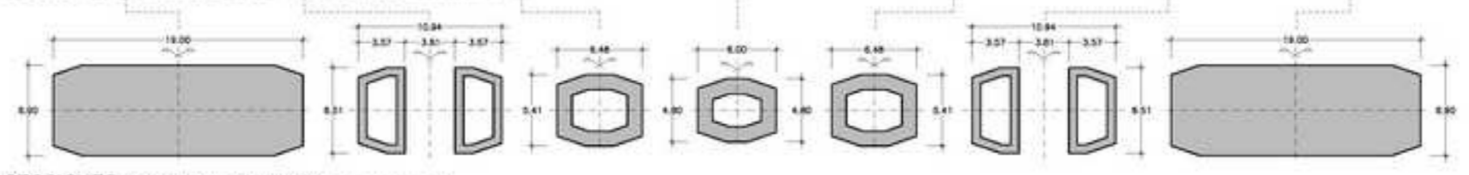

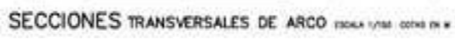

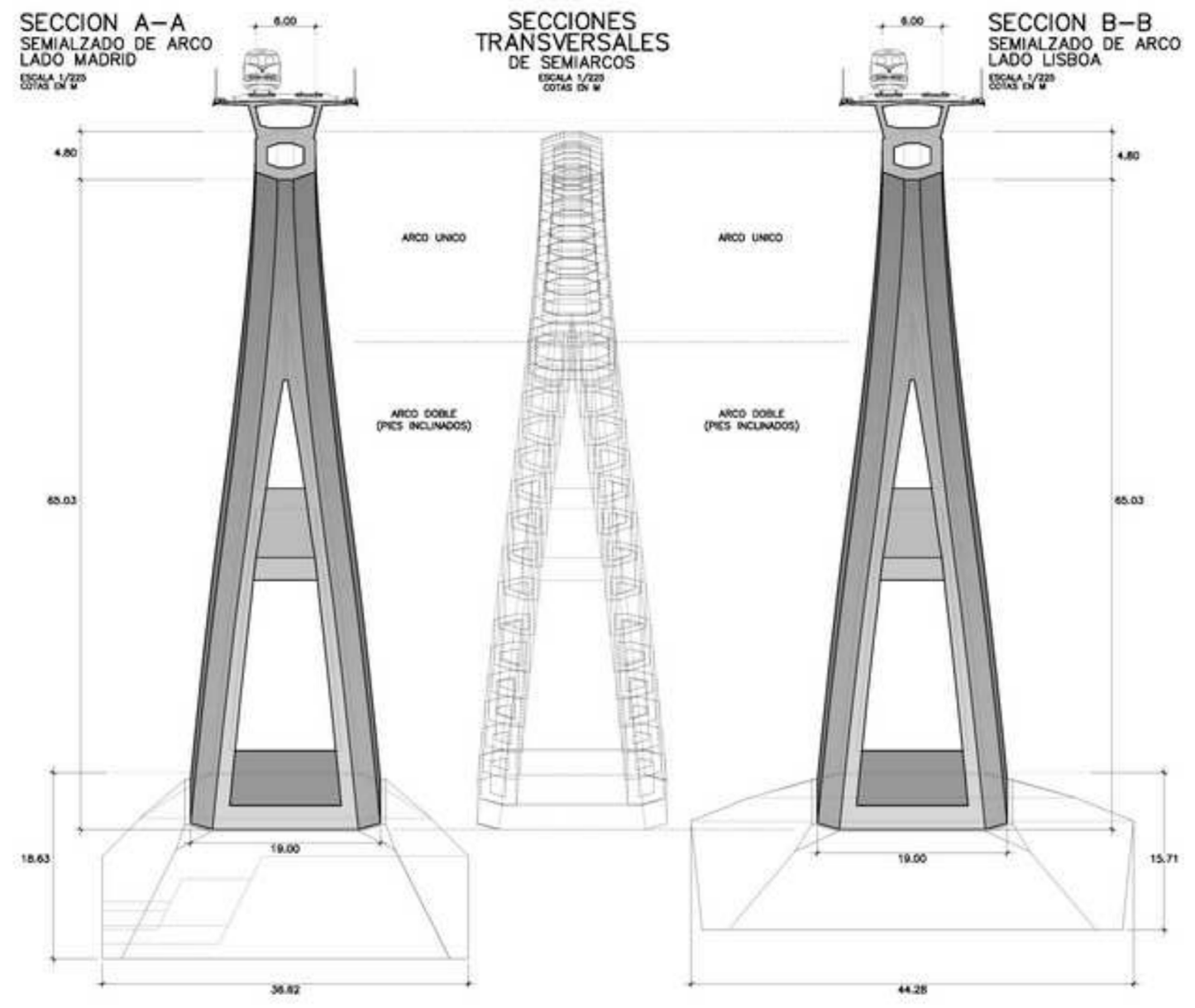

Figura 6. Geometría del arco de Almonte. 

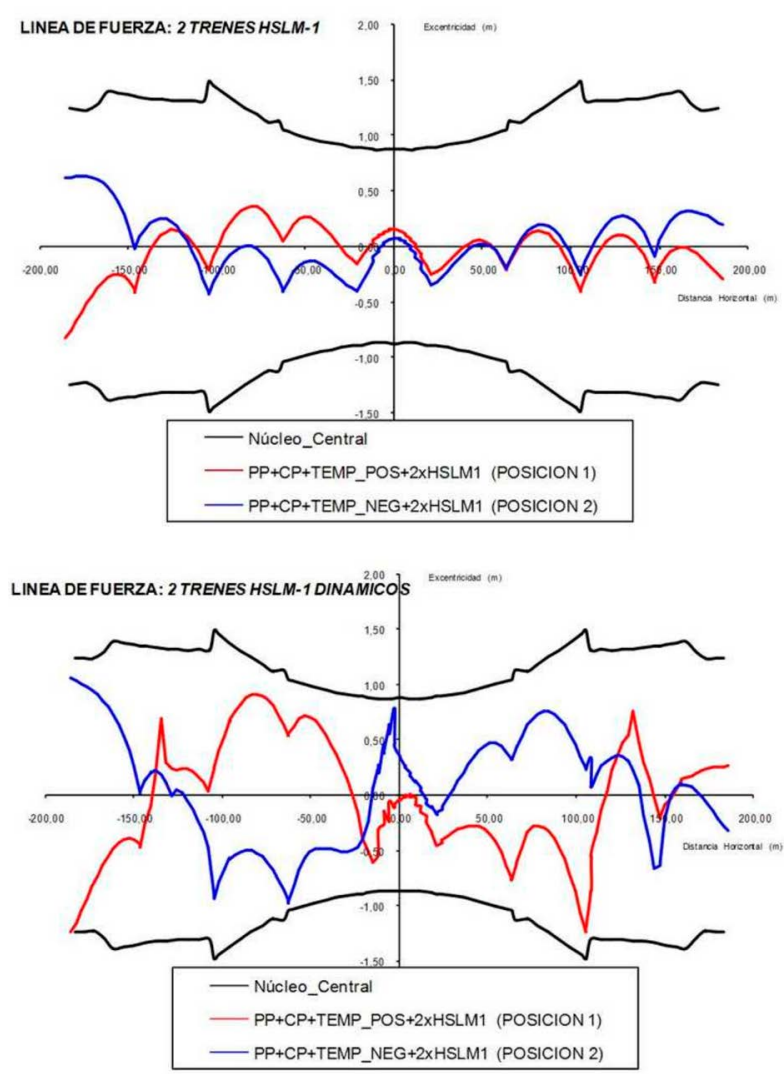

Figura 7. Líneas de fuerza a lo largo del arco para cargas ferroviarias sin y con efectos dinámicos respecto al núcleo central.

De esta forma el material constituyente del arco está exento de tracciones y, al no fisurar el hormigón, se garantiza la durabilidad del puente con un mantenimiento mínimo además de economizar en las cuantías de acero que son las que, dado el caso, deben resistir las tracciones.

En el caso de los ferrocarriles de alta velocidad, la búsqueda de la directriz antifunicular en los grandes arcos ferroviarios impone una nueva realidad en la metodología empleada hasta la fecha en el encaje de la misma: en el ferrocarril los trenes de carga correspondientes al material móvil suponen importantes deformaciones y, por tanto, esfuerzos de flexión de valor absoluto considerables [5].

Se convierte por ese motivo en pieza importante del diseño de un puente arco la decisión de si se acepta fisuración en el arco en situaciones de servicio o no. Se da el caso particular de que aceptar una fisuración limitada del arco en ciertas situaciones de servicio, hace difícil y encarece el cumplimiento de las deformaciones límites para el servicio de la estructura, ya que las fisuras degradan la rigidez de la estructura.

Es decir, cuando esta degradación de la rigidez ocurre en un arco, los movimientos con los que responde a las cargas son mayores, y para lograr unas flechas admisibles adecuadas al uso ferroviario sería necesario dimensionar el puente para las inercias en fisuración, o sea, sería necesario aumentar la cantidad de material y el canto en comparación a si se pudiese evitar la fisuración. Es por eso que en los puentes arco de ferrocarril es aconsejable un criterio general de evitar la fisuración ante sobrecargas en situaciones de servicio aprovechando las herramientas en juego.
Hay que tener en cuenta también la influencia de los efectos dinámicos sobre los esfuerzos de sobrecargas sobre la envolvente de excentricidades que debe situarse dentro del núcleo central del arco (figura 7).

Para las situaciones de estado límite último (ELU), resulta prácticamente inevitable evitar la fisuración con las solicitaciones producidas por las cargas mayoradas y las resistencias de los materiales minoradas, por lo que todas las comprobaciones referidas del comportamiento global del arco y la estabilidad del mismo en situaciones de ELU deben hacerse teniendo en cuenta los efectos de la fisuración. Sin embargo, optimizar los momentos nucleares, es una condición necesaria para minimizar la cuantía de armadura precisa en ELU.

Así, es necesario seleccionar la directriz que minimiza los esfuerzos de flexión en el arco teniendo en cuenta el proceso constructivo y estos efectos dinámicos, y de forma simultánea seleccionar el canto más adecuado, que define su rigidez y la altura de su núcleo central. Este proceso de cálculo es necesariamente iterativo, no lineal y considerando todas las fases de la ejecución, lo que lo hace muy complejo, pero sin embargo fundamental para el objetivo de durabilidad buscado.

Otros análisis que en esta estructura resultan fundamentales para evaluar su comportamiento incluyen la influencia de la no linealidad del material y geométrica. Estos comprenden la evaluación de la retracción y fluencia, el control de la fisuración del hormigón durante las fases de la secuencia de construcción y en servicio con el puente terminado, la no linealidad de las ecuaciones constitutivas del acero y del hormigón. Además, la consideración de la no linealidad geométrica, y los efectos de segundo orden considerando imperfecciones de montaje. Así como también el cálculo dinámico y control de la deformabilidad de la estructura para cumplir los criterios de funcionalidad para la seguridad y confort de los usuarios.

Todas estas cuestiones, se desarrollan en artículos específicos de este monográfico.

\section{7. COMPORTAMIENTO FRENTE A VIENTO Y ENSAYOS DE TÚNEL DE VIENTO}

En estructuras de luz superior a $200 \mathrm{~m}$, el comportamiento de la estructura frente a las cargas de viento es un factor clave en el diseño y de obligado estudio. El canto del arco debe ser lo más reducido posible para presentar una menor superficie expuesta al viento, y la forma de la sección transversal debe optimizarse en base al conocimiento de las propiedades del flujo del viento con un perfilado efectivo como para establecer un arco compacto y opaco al viento. Sin embargo, además debe poseer la inercia suficiente para hacer frente a los momentos flectores concomitantes provenientes de las cargas de tráfico.

El resultado de todo el análisis anterior conduce a que el viaducto sobre el río Almonte tenga una sección hueca, que cumple los requisitos de compacidad y, por tanto, perturbación menor del flujo aéreo con el menor peso. La sección es de forma octogonal con chaflanes pronunciados que afilan su perfil para los vientos de dirección horizontal. El análisis en túnel de viento sobre modelo reducido ha confirmado la bondad del diseño. 

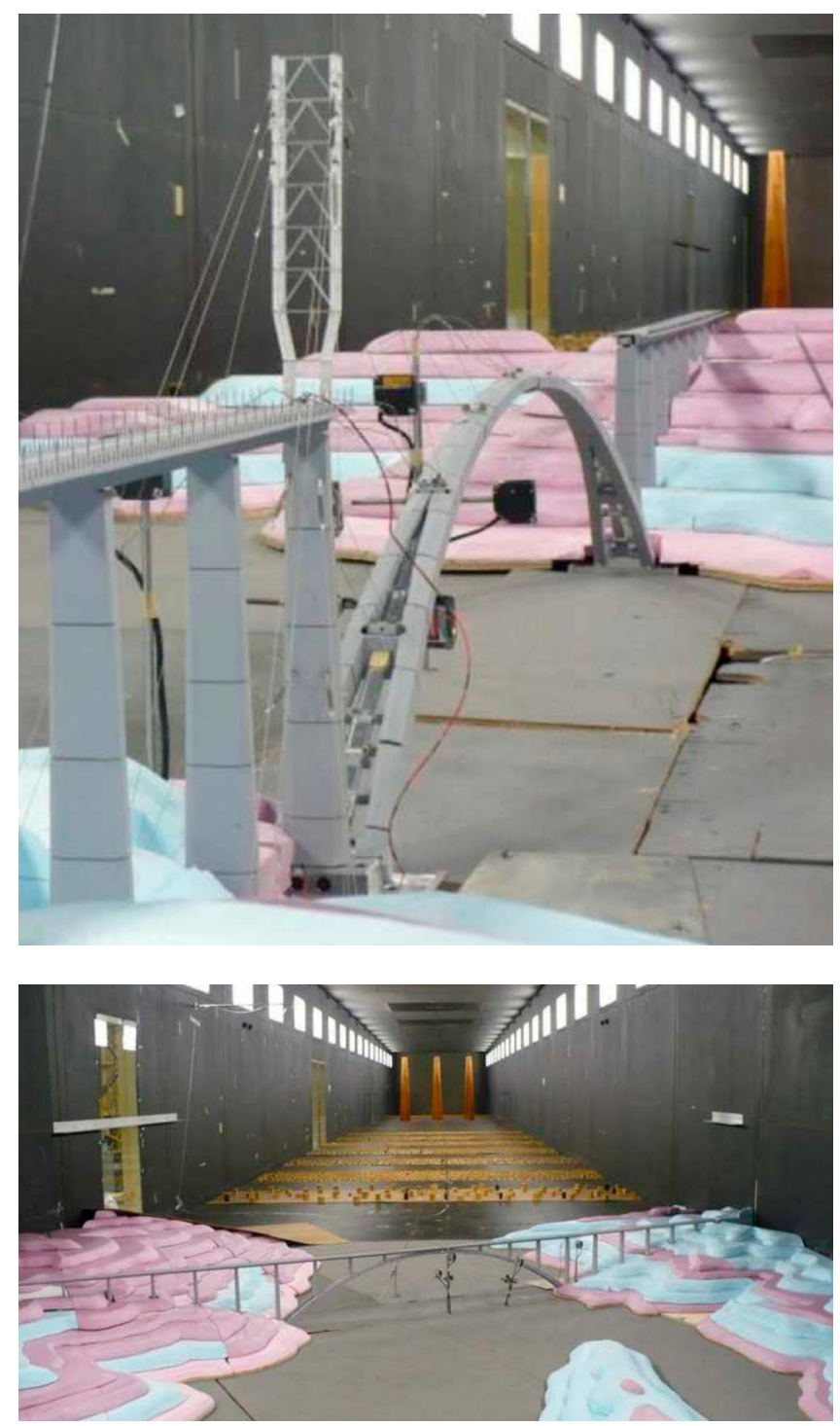

Figura 8. Túnel de viento durante las fases constructivas y en situación final (Fuente: Oritia \& Boreas).

Se han realizado modelos seccionales del arco y del tablero, y modelos globales de túnel de viento a escala 1:210 del viaducto tanto en situación de estructura completa como en construcción en situación de voladizo con el atirantamiento provisional, teniendo en cuenta la orografia del terreno (figura 8).

La medición de velocidades de viento y aceleraciones de la estructura real durante la construcción permitió evaluar con un nivel de precisión sin precedentes el comportamiento estimado en los estudios de viento llevados a cabo en la Universidad de Western Ontario (Canadá) [6].

\section{8.}

\section{DESARROLLO DE LAS OBRAS}

La construcción del puente comenzó en julio de 2011 y su finalización se alcanzó a finales de 2016, si bien actualmente se eliminan las penínsulas provisionales del embalse y se

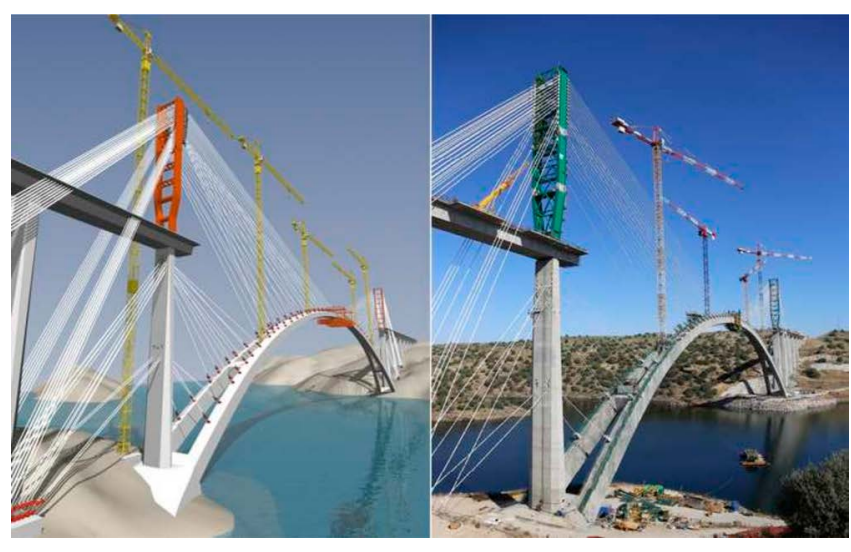

Figura 9. Comparativa entre el procedimiento constructivo desarrollado durante el proyecto, y la imagen real durante la ejecución de las obras.

restablecen las laderas para naturalizar en lo posible todos los movimientos de tierra que conllevó la construcción.

El procedimiento de ejecución principal para el arco es el de avance en voladizo con atirantamiento provisional, siguiendo la misma secuencia considerada en el proyecto de construcción (figura 9).

La construcción comenzó con la ejecución de las cimentaciones y la erección de alzados de estribo y pilas con encofrado trepante. El tablero de ambos accesos se ejecuta con autocimbra hasta alcanzar el vano anexo al tramo de tablero sobre el arco. Para la materialización de los puntos de retenida de los cables de contrarresto del atirantamiento provisional se realizan anclajes al terreno en las cimentaciones de las pilas anexas a las que llegan los tirantes. Estos anclajes se ensayan y se instrumentan para su control.

La ejecución mediante avance en voladizo del arco requiere la construcción de unos carros de avance de encofrado de gran complejidad que permiten variar todas las dimensiones de la sección para adaptarse a sus formas variables. Los carros son dos por arco en los pies inclinados curvos en arranques para luego combinarse y conformar un carro único en la zona central del arco (figura 10).

Cada dovela tiene una longitud en proyección en planta de aproximadamente $6 \mathrm{~m}$, lo que supone longitudes desarrolladas de entre 6.80 y $6.20 \mathrm{~m}$.

Los tirantes se anclan en las primeras dovelas del arco en la pila sobre el arranque del arco, pero a partir de una determinada dimensión del voladizo su dimensión resulta insuficiente y es necesaria una torre de atirantamiento provisional.

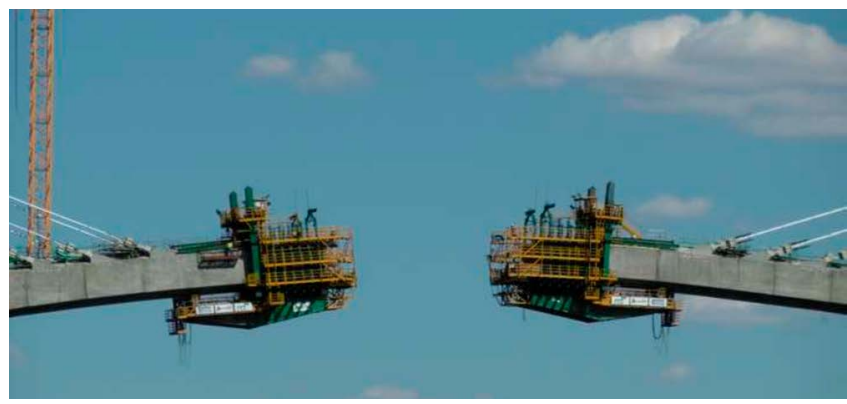

Figura 10. Ejecución de las últimas dovelas del arco. 


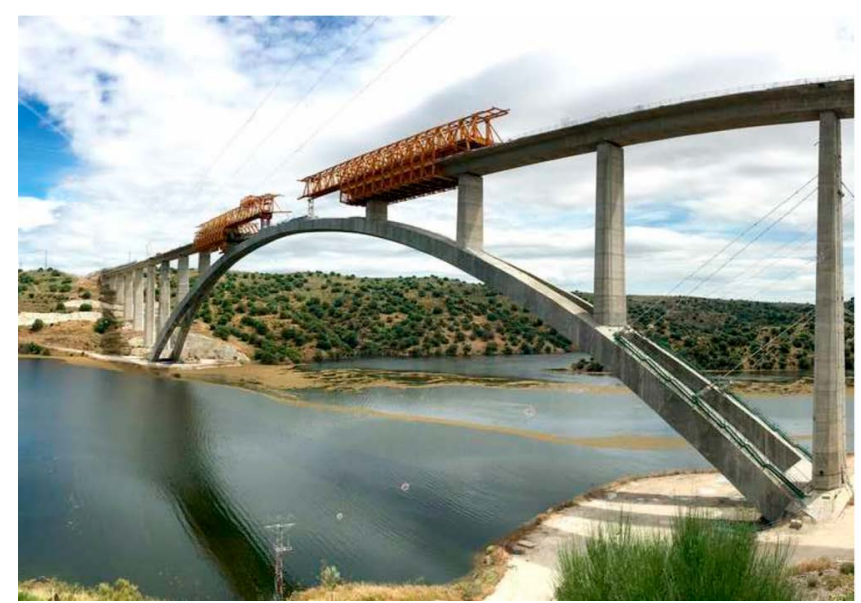

Figura 11. Ejecución de los últimos vanos del tablero.

Esta torre de atirantamiento provisional, desarrollada por los servicios técnicos de FCC, constituye una estructura en sí misma, con una altura de más de $50 \mathrm{~m}$ sobre el tablero y articulada en la base. Su montaje se realiza mediante giro de abatimiento con respecto a esta rotula inferior.

Una vez finalizada la construcción del arco, se descienden los carros de avance y vuelven a entrar en escena las autocimbras, con el fin de ejecutar los vanos del tablero restantes sobre las pilastras que apoyan directamente sobre el arco (figura 11).

Todo el proceso de construcción ha sido monitorizado con una instrumentación con medición continua que incluía clinómetros, acelerómetros, galgas extensométricas, células de carga en tirantes de retenida, medidas topográficas automatizadas y medidas meteorológicas de viento y temperatura [7].

Registrándose todos estos valores para su evaluación, se permitió detectar desplazamientos o cambios en las frecuencias de vibración de la estructura que alertasen de cualquier cambio o anomalía en el comportamiento de la misma. Actualmente, se dispone de instrumentación definitiva que permitirá evaluar de manera inmediata el comportamiento de la estructura en servicio.

\section{9.}

\section{CONCLUSIONES}

El estado actual y los avances de la técnica y la construcción, han permitido proyectar y afrontar la ejecución de este gran arco de hormigón, un auténtico reto que sitúa a la ingeniería y construcción española en completa vanguardia. Entre los aspectos más destacados, hay que señalar los hormigones de altas prestaciones, las herramientas de cálculo avanzado no lineal y evolutivo, la modelización aerolástica, y los tratamientos semiprobabilísticos normativos, como elementos clave para el diseño y la validación de la garantía estructural del viaducto de Almonte.

El uso conjunto e intensivo de todos ellos ha permitido superar los rangos de luz que hasta ahora se habían establecido para puentes ferroviarios de alta velocidad, resaltando la importancia de las elevadas sobrecargas, la influencia de la amplificación dinámica de sus efectos y del comportamiento no lineal tanto geométrico como del material para valorar con precisión los niveles de seguridad con los que se dimensiona y se construye esta estructura.

Desde su aparente sencillez, el diseño del puente da solución, simultáneamente, a los múltiples requerimientos tanto funcionales, como estructurales y medioambientales del difícil problema de cruce. Las soluciones aparentemente más sencillas a un problema complejo, en el diseño de puentes y en muchas otras disciplinas, suelen ser las más difíciles de alcanzar.

10.

\section{AGRADECIMIENTOS}

Destacar la capacidad, formación y dedicación del equipo de IDOM y Arenas \& Asociados que hizo posible, en tiempo récord, el proyecto de este puente.

Al trabajar en el resto del mundo y en contacto o colaboración con ingenierías de tamaño mucho mayor, se contrasta mejor la capacidad de organización y trabajo en equipo que se ha llegado a alcanzar en nuestras ingenierías. El equipo y tiempo empleado para desarrollar este puente de récord, sin querer hablar de honorarios, se muestra casi ridículo con lo equivalente en el contexto internacional.

Gracias por ello a Emilio Merino, Ysabel Guil, Javier Martínez, Héctor Beade, Santiago Guerra, Pablo Alfonso, Marianela García, Juan Ruiz, Javier Fernández, Miguel Sacristán, Diego González, Pablo Cuesta, Eduardo Rojo, Fátima Castillo, y resto de profesionales sin los que el éxito del proyecto no habría sido posible.

\section{Referencias}

[1] TORRES, J., SANTAMARÍA, A.J., ARENAS, J.J., DÍAZ DEL VALLE, J. y LORENZO, D., "Tipología. Arcos. Funicularidad". Servicio de Publicaciones de la E.T.S. de Ingenieros de Caminos, Canales y Puertos. Universidad de Cantabria. 1988.

[2] FERNÁNDEZ CASADO, C., "Cálculo de arcos". Editorial DOSSAT, S.A.

[3] ARENAS DE PABLO, J.J., "El puente, pieza esencial del mundo humanizado". Lección inaugural del curso académico 1982-83 de la Universidad de Santander. Secretariado de Publicaciones.

[4] CAPELlÁN, G., BEADE, H., ARENAS, J.J., GARCÍA, P. y MEANA, I., "Diseño del puente arco de alta velocidad sobre el río Almonte en el Embalse de Alcántara". VI Congreso de ACHE. Madrid, pon 007.

[5] ARENAS, J.J., CAPELLÁN, G., BEADE, H., MERINO, E., MARTÍNEZ J., GUIL, Y., y GARCÍA, P., "Viaducto sobre el río Almonte en el Embalse de Alcántara. Comportamiento y cálculo". VI Congreso de ACHE. Madrid, pon 058.

[6] TERRÉS, J.M., MANS, C., KING, P.J.C., CAPELLÁN, G. y BEADE H., "Estudio experimental del efecto del viento en un puente arco de $384 \mathrm{~m}$ de luz en fases de servicio y constructiva". V Congreso de ACHE, Barcelona, pon 650 .

[7] CAPELLÁN, G., MARTÍNEZ, J., MERINO, E., GARCÍA, P. y JIMÉNEZ, P., "Viaducto de Almonte. Diseño y control de construcción". VII Congreso de ACHE. A Coruña, pon 318. 

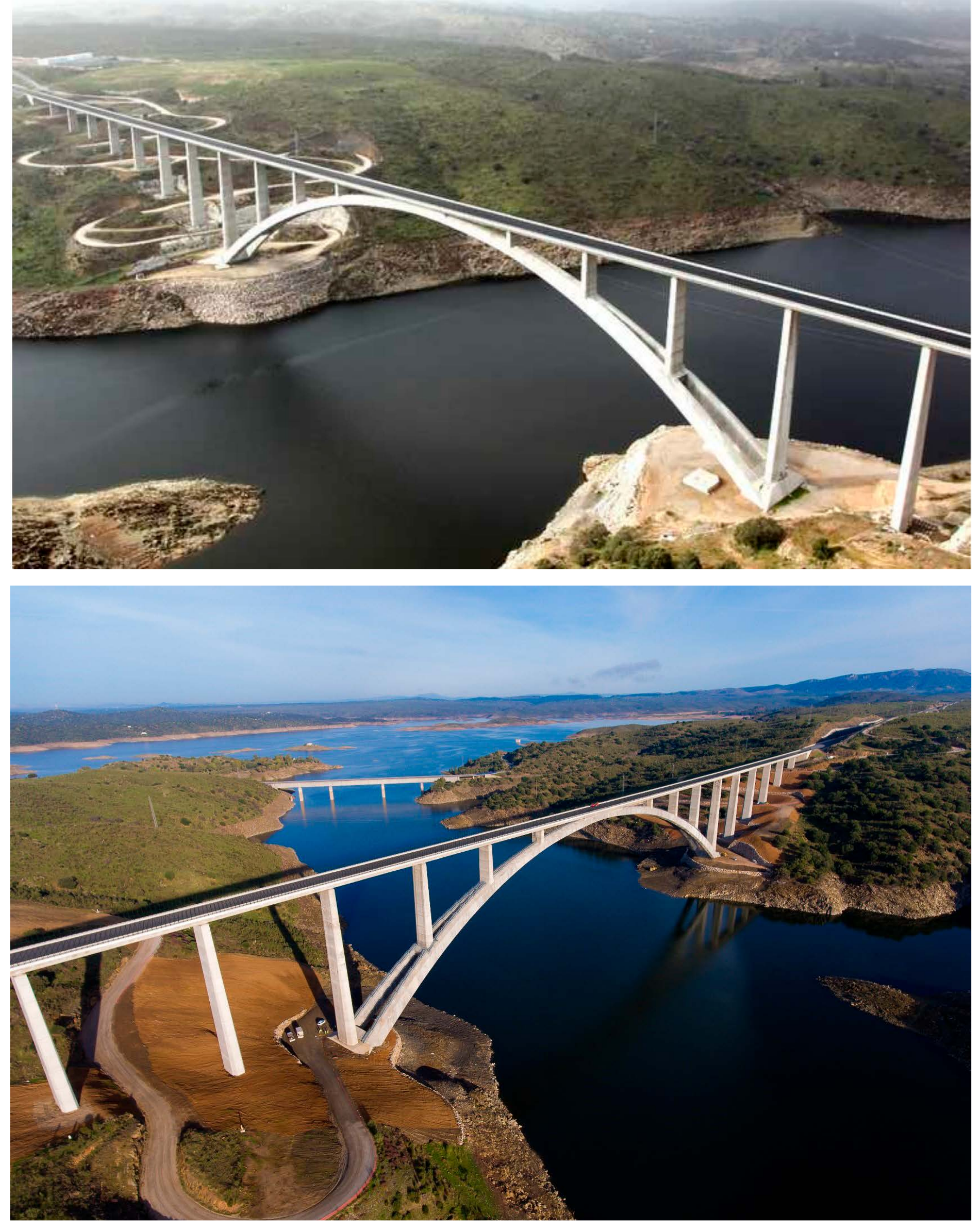

Figura 12. Vistas aéreas del viaducto de Almonte (Fuente: FCC). 